Original article

\title{
Evaluation of monocyte subsets and markers of activation in leprosy reactions
}

\author{
Mayume Shibuya ${ }^{a}$, Giovana Bergheme ${ }^{a}$, Sara Passos ${ }^{\mathrm{a}, \mathrm{b}}$, Ivonete Queiroz ${ }^{\mathrm{a}}$, \\ Jamile Rêgo $^{\text {a, b }}{ }^{\text {, Lucas P. Carvalho }}{ }^{\text {a, b, c }}$, Paulo R.L. Machado ${ }^{\text {a, b, * }}$ \\ a Programa de Pós-Graduação em Ciências da Saúde, Federal University of Bahia, Salvador, Bahia, Brazil \\ b Immunology Service, Hospital Universitário Professor Edgard Santos, Federal University of Bahia, Salvador, Bahia, Brazil \\ c Institute Oswaldo Cruz Foundation, Salvador, Bahia, Brazil
}

\section{A R T I C L E I N F O}

Article history:

Received 26 April 2018

Accepted 17 October 2018

Available online $\mathrm{xxx}$

\section{Keywords:}

Leprosy

Leprosy reactions

Monocytes

Co-stimulatory molecules

\begin{abstract}
A B S T R A C T
Understanding host immune pathways associated with tissue damage during reactions are of upmost importance to the development of immune intervention strategies. The participation of monocytes in leprosy reactions was evaluated by determining the frequency of monocyte subsets and the degree of cellular activation through the expression of MHCII and the co-stimulatory molecules CD40, CD80, CD86. Leprosy subjects with or without reactions were included in this cross-sectional study. Peripheral blood mononuclear cell were isolated and stained ex vivo to determine monocyte subsets and the degree of cellular activation by flow cytometry. Intermediate monocytes were increased in leprosy patients with reactions when compared to patients without reactions. Although no difference was detected in the frequency of monocyte subsets between type 1 and 2 reactions, the expression of CD80 was increased in monocytes from patients with type 1 reactions and CD40 was higher in paucibacillary subjects presenting type 1 reactions. Moreover, CD86 and MHC II expression were higher in intermediate monocytes when compared to the other subsets in leprosy reaction types 1 and 2. Intermediate monocyte activation with CD86 and MHCII expression is involved with both type 1 and 2 reactions, whereas CD80 and CD40 expression is related to type 1 reactions.
\end{abstract}

(c) 2018 Institut Pasteur. Published by Elsevier Masson SAS. All rights reserved.

\section{Introduction}

Leprosy affects more than 200,000 persons yearly in the world with 26,395 new cases registered in Brazil in 2015 [1]. The wide clinical presentation of leprosy depends almost exclusively on the host immune response against Mycobacterium leprae [2,3]. Whereas a strong cell-mediated immunity is associated with a negative bacillary index (BI) and few localized cutaneous lesions with raised borders in the tuberculoid pole (TT), at the opposite pole of the spectrum, patients with lepromatous leprosy (LL) present disseminated infection due to the absence of specific cellmediated immunity against $M$. leprae along with multiple

\footnotetext{
* Corresponding author. Rua João das Botas, $\mathrm{S} / \mathrm{N}^{\circ}, 5^{\circ}$ andar, Hospital Universitário Professor Edgard Santos, Serviço de Imunologia, Salvador, Bahia, 40.110-160, Brazil.

E-mail addresses: mayshibuya@hotmail.com (M. Shibuya), gbergheme@hotmail. com (G. Bergheme), saratpassos@hotmail.com (S. Passos), ivonetesq@hotmail.com (I. Queiroz), jamileao@hotmail.com (J. Rêgo), carvalholp76@gmail.com (L.P. Carvalho),19pmachado@gmail.com, prlmachado@uol.com.br (P.R.L. Machado).
}

disseminated nodules and infiltrated lesions with high BI [2,3]. Besides these opposite poles, most patients are classified as presenting borderlines forms of leprosy according to variable degrees of cellular immune response against $M$. leprae and clinical manifestations [4]. Therefore, borderline-tuberculoid (BT) leprosy is characterized by lesions with raised and enlarged borders and a negative or low BI; borderline-borderline (BB) subjects presents several lesions with ill demarcated infiltrated borders and a positive $\mathrm{BI}$; borderline-lepromatous (BL) patients shows disseminated asymmetrical distributed nodules and infiltrated plaques with a positive BI. During the chronic course of leprosy, acute inflammatory reactions can occur before, during or after treatment with multidrug therapy (MDT), leading to peripheral nerve function impairment (NFI) and long-term disabilities. Type 1 reaction (T1R) or reversal reaction is due to an increased cellular hypersensitivity mediated by specific T-cells against M.leprae antigens in the skin and peripheral organs, being more common in borderline subjects. Type 2 reaction (T2R) or erythema nodosum leprosum is associated to immune complex deposits as well as high production of 
inflammatory cytokines including TNF- $\alpha$ in skin, peripheral nerves and internal organs, affecting BL and LL patients [2,3]. According to WHO, leprosy is the leading infectious disease cause of physical disabilities around the world [1]. The development of better therapeutic strategies to control reactions and avoid NFIs is of upmost importance to avoid leprosy burden. It requires a better understanding of the host immune pathways associated with tissue damage during reactions leading to permanent loss of nerve function.

Monocytes differentiate into macrophages in tissues behaving as phagocytic and antigen presenting cells (APCs), with an important contribution not only in the innate immune response, but also in the adaptive immune response by T-cell activation. The fact that macrophages are heavily parasitized by $M$. leprae in the lepromatous pole, along with their capacity to destroy mycobacteria $[3,4]$, highlights their essential contribution to control of the disease. Monocytes may contribute to defense against $M$. leprae by inducing high expression of the GTPase family of M protein, which correlates inversely with the severity of leprosy [5]. Monocytes are divided in three subsets according to the expression of CD14 and CD16: CD14 + CD16- (classical), CD14 + CD16+ (intermediate), and CD14lowCD16++ (nonclassical), with differential functions and properties like antigen presentation, chemokine production and cytokine production [6,7]. In cutaneous leishmaniasis, intermediate monocytes are associated with inflammatory responses and pathology [8]. However, the role of monocyte subsets in leprosy control and reactions remain unknown. The aim of this study was to evaluate the role of monocytes in leprosy reactions by determining the frequency of monocyte subsets and degree of cellular activation through the expression of co-stimulatory molecules CD40, CD80, CD86 and MHC II.

\section{Material and methods}

\subsection{Patients}

Sixty patients classified by the Ridley \& Jopling criteria [4] and also by WHO operational classification [1] as multibacillary (MB) or paucibacillary (PB) according to a positive or negative baciloscopy respectively, were included in this cross-sectional study. A total of 34 leprosy subjects were diagnosed without reactional episodes and 26 patients presented leprosy reactions. Subjects were recruited at the Leprosy Clinics in the Hospital Universitário Prof. Edgar Santos from the Federal University of Bahia and Hospital Dom Rodrigo de Menezes, both reference centers for the treatment of leprosy. All patients were submitted to a complete dermatological and neurological evaluation, skin lesion biopsy and split skin smear. We excluded patients using corticosteroids, thalidomide or immune suppressants, pregnant women, and subjects younger than 18 years old or older than 65 years old.

\subsection{Ex vivo assay}

Heparinized peripheral blood $(20 \mathrm{ml})$ was collected for isolation of peripheral blood mononuclear cells (PBMC) according to standard protocol. Briefly, PBMCs were isolated by concentration gradient with Ficoll-Hypaque. After the process of three washes with $0.9 \% \mathrm{NaCl}$, PBMCs were resuspended in culture with RPMI1640 medium supplemented with $10 \%$ inactivated fetal bovine serum, HEPES buffer and Gentamycin $100 \mathrm{IU} / \mathrm{ml}$. PBMCs were stained ex vivo with following antibodies to determine monocyte subpopulations: anti-CD14 - APC, anti-CD16 - PE and antibodies anti-MHC II - FITC, anti-CD80 - FITC, anti-CD40 - PE, anti-CD86 PerCP-Cy5.5 to assess the degree of cellular activation. After ex vivo protocol the cells were acquired by BD cytometer CANTO II. Cells were gated on monocyte region based on forward and side scatter, and on CD14 + cells. Gates were done based on all fluorescence minus one (FMO).

\subsection{Statistical analysis}

To compare data between two independent continuous variables we used Mann-Whitney test. Analysis among three independent continuous variables was performed using Kruskal-Wallis test and Dunn posttest. The differences were considered significant when $\mathrm{p}<0.05$. GraphPad Prism 5.0 software was chosen (GraphPad Software, San Diego, CA, USA). The flow cytometry data was analyzed using FlowJo software (Tree Star, Ashland, OR, USA).

\section{Results}

\subsection{Patient characteristics}

From the total of sixty leprosy patients, 34 subjects did not present reactions and 26 were diagnosed with reactions. In the group of patients without reactions, the majority were paucibacillary cases. According to the Ridley-Jopling classification [4], eleven had indeterminate leprosy (I), 8 had tuberculoid (TT) form, 3 had borderline tuberculoid (BT), 3 borderline borderline (BB), 3 borderline lepromatous (BL) and 6 lepromatous leprosy (LL). In the group of patients with reactions, 13 presented type 1 reactions (T1R) and 13 were diagnosed with type 2 (T2R). Borderline forms predominated in the T1R group and lepromatous pole in the T2R group. Table 1 shows demographic and clinical data from all subjects.

\subsection{Frequency of monocyte subsets}

We analyzed the frequency of monocyte subsets based on the expression of CD14 and CD16 in leprosy patients with or without reactions. A higher frequency of intermediate monocytes was observed in subjects presenting reactions, whereas non-classical monocytes were more frequent in patients without reactions (Fig. 1). When comparing the T1R group with the T2R group, we found no significant difference regarding monocyte subset frequencies (Fig. 2). In both T1R and T2R, classical and intermediate subpopulations predominate. These data indicate that intermediate monocytes, which are characterized by the production of inflammatory cytokines, are involved in leprosy reactions.

\subsection{Co-stimulatory molecules and MHC II expression by monocytes CD14+}

CD80 expression was higher in patients with T1R when compared to T2R, suggesting that the up-regulation of CD80 may be associated with macrophage activation and production of proinflammatory cytokines in T1R. CD40 Mean fluorescence intensity (MFI) expression did not differ between the T1R and T2R but it was higher in PB subjects with T1R when compared with PB patients without T1R (Fig. 3).

CD86 is a co-stimulatory molecule that induces T cell activation and its overexpression is associated with inflammatory responses. We found that higher expression of CD86 in intermediate than in classical and non-classical monocytes in both T1R and TR2 patients (Fig. 4). No difference was found when comparing CD86 expression in PB patients with or without T1R as well as between MB subjects with or without T2R (data not shown).

Expression of MHC II did not differ between PB subjects with or without T1R and also between MB patients with or without T2R (data not shown). Intermediate monocytes expressed more MHC II 
Table 1

Demographic and clinical data from leprosy patients regarding presence or absence of reactional episodes.

\begin{tabular}{|c|c|c|c|c|}
\hline \multirow[t]{2}{*}{ Group (n) } & Age (mean $\pm \mathrm{SD})$ & \multirow[t]{2}{*}{ Male:Female Ratio } & \multirow[t]{2}{*}{ Ridley-Jopling classification ${ }^{a}$} & \multirow[t]{2}{*}{$\mathrm{BI}^{\mathrm{b}}$} \\
\hline & (years) & & & \\
\hline Without reactions (34) & $42 \pm 13.5$ & $16: 18$ & $\begin{array}{l}11 \mathrm{I} \\
8 \mathrm{TT} \\
3 \mathrm{BL} \\
3 \mathrm{BT} \\
3 \mathrm{BB} \\
6 \mathrm{LL}\end{array}$ & $3 \pm 1.94$ \\
\hline Type 1 reaction (13) & $44 \pm 12.2$ & 7:06 & $\begin{array}{l}6 \mathrm{BT} \\
5 \mathrm{BB} \\
2 \mathrm{BL}\end{array}$ & $1 \pm 0.97^{c}$ \\
\hline Type 2 reaction (13) & $34 \pm 8.4$ & 9:04 & $\begin{array}{l}6 \mathrm{BL} \\
7 \mathrm{LL}\end{array}$ & $3 \pm 1.2$ \\
\hline
\end{tabular}

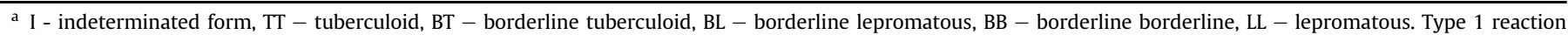
(reversal reaction) and type 2 reaction (erythema nodosum leprosum).

b BI - Bacillary index.

c MB cases.

A.
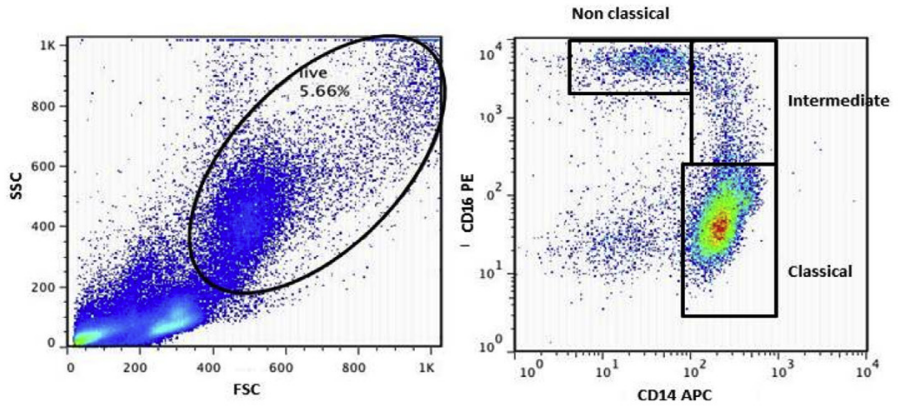

B.

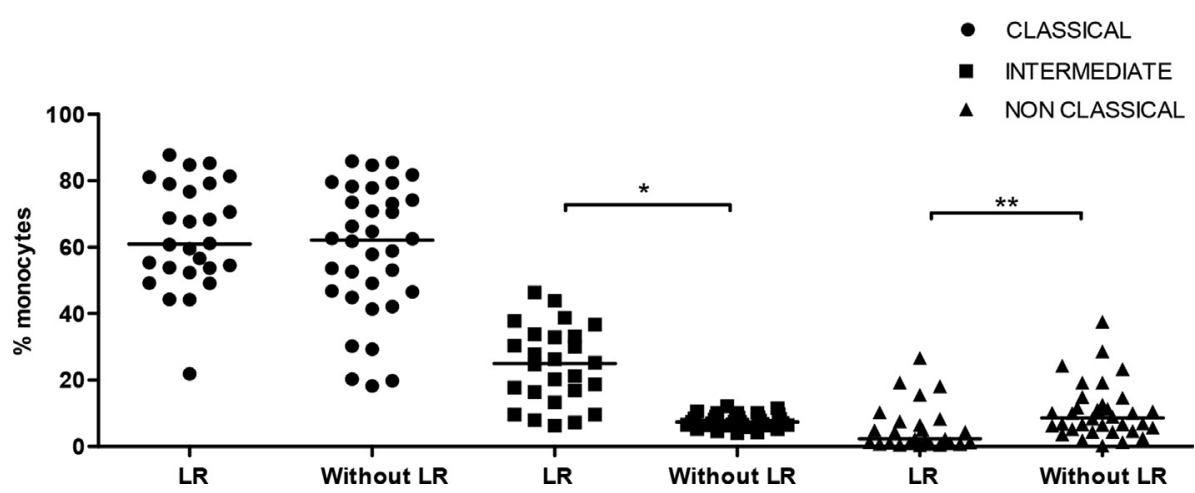

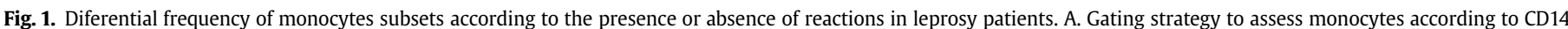

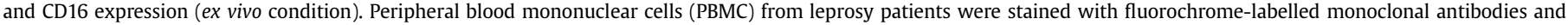

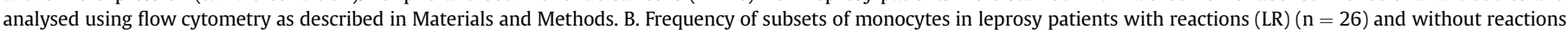

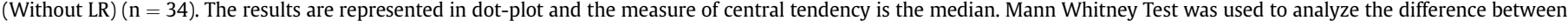
the two groups in the different sub-populations of monocytes, ${ }^{*} \mathrm{P}=0.0001 ;{ }^{* *} \mathrm{P}=0.001$.

than the other populations of monocytes in subjects with T1R and T2R (Fig. 5). The higher expression of MHC II and CD86 in intermediate monocytes is consistent with the inflammatory phenotype of this subpopulation of monocytes.

\section{Discussion}

An efficient immune response against $M$. leprae infection is associated with Th1 response and cytotoxicity halting the progression of the disease [9]. On the other hand, activation of a Th2 response inhibits granuloma formation allowing $M$. leprae multiplication [10]. Monocytes play an important role in granuloma formation and in the defense mechanisms against $M$. leprae infection [11], but its participation in the inflammatory pathways associated with leprosy reactions remain ignored. In the present study we determined the frequency of monocyte subpopulations in leprosy patients with and without reactions and assessed the expression of MHC II and co-stimulatory molecules in these cells.

The circulating monocyte population is phenotypically and functionally heterogenic. Most studies show that classical and intermediate populations are pro-inflammatory and secrete high levels of TNF- $\alpha$, whereas, non-classical monocytes are associated 


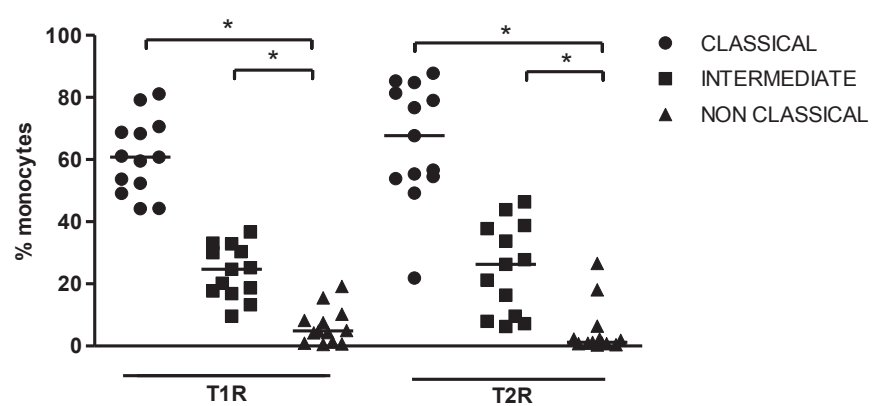

Fig. 2. Frequency of monocytes subsets in the different types of reaction in leprosy. Frequency of monocytes sub-populations in patients with type 1 reaction (T1R) $(\mathrm{n}=13)$ and type 2 reaction $(T 2 R)(\mathrm{n}=13)$ analyzed by flow citometry. The results are represented in dot-plot and the measure of central tendency is the median. Nonparametric Kruskal-Wallis and Dunns post test were used to analyze the differences between groups, ${ }^{*} \mathrm{P}=0.001$.

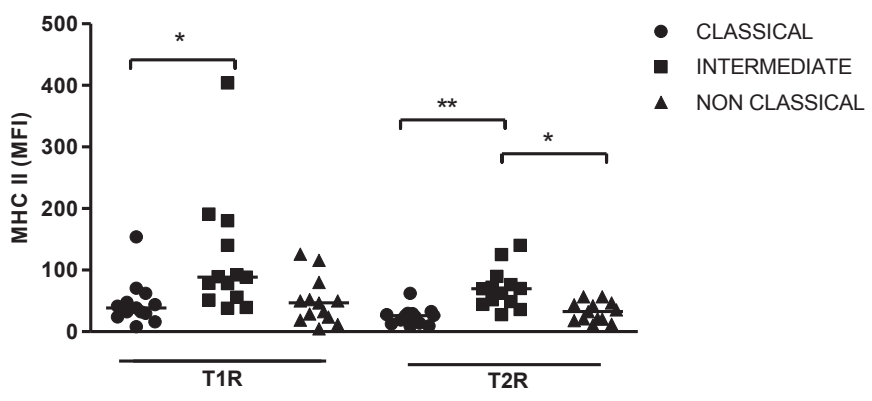

Fig. 5. Expression of MHCI in subsets of monocytes from patients with type 1 and type 2 leprosy reactions. Mean fluorescence intensity (MFI) of sub-populations of monocytes in patients with leprosy reaction $(\mathrm{LR})(\mathrm{n}=26)$ and without leprosy reaction (Without LR) $(\mathrm{n}=34)$. The results are represented in dot-plot and the measure of central tendency is the median. Non-parametric Kruskal-Wallis and Dunns posttest were used to analyze the difference between groups ${ }^{*} \mathrm{P}<0.0001 ;{ }^{* *} \mathrm{P}=0.004$.
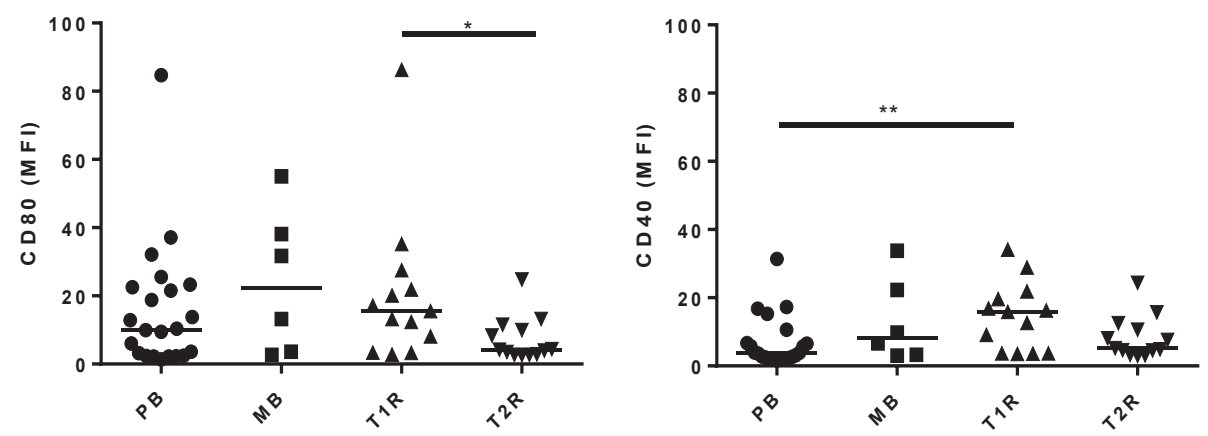

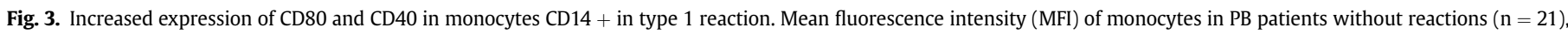

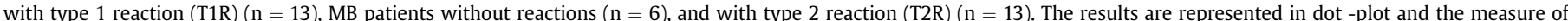

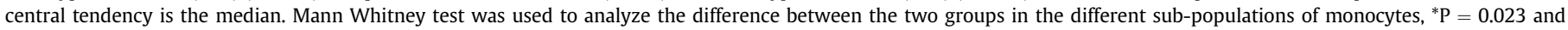
${ }^{* *} \mathrm{P}=0.0075$.

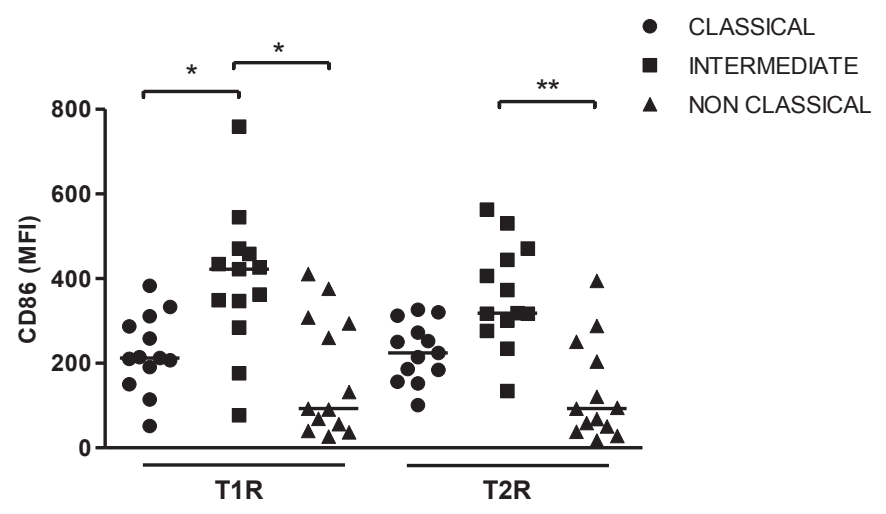

Fig. 4. Expression of CD86 in monocytes subsets from patients with $T 1 R$ and $T 2 R$ Mean fluorescence intensity (MFI) of sub-populations of monocytes in patients with type 1 reaction $(T 1 R)(n=13)$ and type 2 reaction $(T 2 R)(n=13)$. The results are represented in dot-plot and the measure of central tendency is the median. Nonparametric Kruskal-Wallis and Dunns post test were used to analyze the differences between groups $* \mathrm{P}=0.001$ and ${ }^{* *} \mathrm{P}=0.0002$.

with wound healing and inflammation resolution. Thus, data accumulated on the literature show the participation of intermediate monocytes in the pathogenesis of many conditions, such as rheumatoid arthritis [12], asthma [13] and cutaneous leishmaniasis $[14,15]$. The main contribution of intermediate monocytes to the pathogenesis of these diseases is through pro-inflammatory cytokine secretion and increased ability to present antigen and prime $\mathrm{T}$ cells [16]. In the present work we found an increased frequency of intermediate monocytes in patients with leprosy reactions when compared to patients without reactions; however, no difference was observed in the frequency of monocyte subsets among T1R and T2R individuals. These results suggest that intermediate monocytes may contribute to the pathogenesis of reactions in the same manner, independent of the type of reaction. Inflammatory process occurs in both types of reactions and intermediate monocytes may contribute to inflammation by efficiently presenting antigen to $\mathrm{T}$ cells and secreting pro-inflammatory mediators like TNF- $\alpha$, IL- $1 \beta$ and IL- 6 that are associated with leprosy reactions. Nevertheless, future studies need to be performed to determine whether intermediate monocytes differ functionally in T1R, T2R and non-reactional leprosy patients.

Expression of co-stimulatory molecules, CD80 and CD86, in antigen presenting cells is required for T cell activation and survival. Conflicting data regarding the role of CD80 in leprosy have been documented. These works show either that CD80 expression is associated with the decreased immune response observed in lepromatous patients, or suggest that increased expression of CD80 may be a predictor of reaction states [17-19]. We show here that monocytes from T1R patients express more CD80 than those from T2R individuals. Differences in cytokines produced by patients with T1R and T2R may explain our result. For instance, it is known that IFN-gamma induces CD80 up-regulation [20]. Immunopathology of T1R is more associated with Th1 immune responses and macrophage activation along with production of pro-inflammatory 
cytokines, what may explain the up-regulation of CD80 in these patients. Conversely, increased CD80 expression may be a consequence of a regulatory mechanism [21]. The dynamics of expression differs between CD80 and CD86 molecules, being that CD86 is expressed early after infection while CD80 is usually expressed in a later phase of the immune response [22]. The delayed expression of $\mathrm{CD} 80$ is in fact associated with regulation of the immune response as this molecule has more affinity for CTLA-4 than CD86. In leprosy patients, it has been documented that co-stimulatory molecules CD86 and CD28 are constitutively expressed by APC and T cells, respectively, while CD80 and CTLA-4 are expressed only 24-48 h after activation of the APC and T cells [23,24]. Thus, the upregulation of $\mathrm{CD} 80$ in T1R individuals may also be a regulatory mechanism due to the increased inflammatory response observed in these individuals.

We have found a higher expression of CD40 in PB patients presenting T1R when compared with PB subjects without reaction but no difference in MB patients with or without T2R. The CD40 interaction with its ligand CD154 is important to drive IL-12 production and development of cellular immunity against intracellular pathogens, but it is also involved in granulomatous inflammation [25]. Therefore, CD40 expression by monocytes in leprosy may be involved not only with protection mechanisms against $M$. leprae, but also in the development of T1R when at high levels.

CD86 and MHC II expression are associated with inflammatory responses and $\mathrm{T}$ cell activation. Although we did not find differences in the expression of CD86 and MHC II between patients with or without reactions, these molecules are expressed mainly by intermediate monocytes in both T1R and T2R.

In summary, intermediate monocytes are activated in leprosy reactions, and may play an important role in tissue damage. CD86, MHCII, CD80 and CD40 overexpression are associated with its activation, and may serve as biomarkers for both T1R and T2R (CD86, MHCII), or T1R (CD80 and CD40). The increase of these molecules in the sera of patients before the development of reactions could be a useful biomarker to predict the development of these reactions. Biomarkers for development of leprosy reactions will indicate risk groups and allow a more careful follow-up and medical care to prevent reactions or initiate an early and more effective treatment. In this scenario, these molecules should also be evaluated as possible targets for immunotherapy of leprosy reactions in the future.

\section{Conflict of interest}

The authors declare that they have no competing interests.

\section{Acknowledgements}

This work was supported by a grant from the Instituto Nacional de Ciência e Tecnologia em Doenças Tropicais (INCT-DT), Brazil.

\section{References}

[1] WHO - World Health Organization. Leprosy. http://www.who.int/lep/en/ Accessed 3 March 2017
[2] Ramos-e-Silva M, Rebello PF. 2001. Leprosy. Recognition and treatment. Am J Clin Dermatol 2001:203-11.

[3] Modlin RL. The innate immune response in leprosy. Curr Opin Immunol 2010;22:48-54.

[4] Ridley DS, Jopling WH. Classification of leprosy according to immunity. A fivegroup system. Int J Lepr Other Mycobact Dis 1966;34:255-73.

[5] Yang D, Chen J, Zhang L, Cha Z, Han S, Shi W, et al. Mycobacterium leprae upregulates IRGM expression in monocytes and monocyte-derived macrophages. Inflammation 2014:1028-34.

[6] Ziegler-Heitbrock L, Ancuta P, Crowe S, Dalod M, Grau V, Hart DN, et al. Nomenclature of monocytes and dendritic cells in blood. Blood 2010;116: e74-80.

[7] Zawada AM, Rogacev KS, Rotter B, Winter P, Marell RR, Fliser D, et al SuperSAGE evidence for CD14 ++ CD16 + monocytes as a third monocyte subset. Blood 2011;118:e50-61.

[8] Passos S, Carvalho LP, Costa RS, Campos TM, Novais FO, Magalhaes A, et al. Intermediate monocytes contribute to pathologic immune response in Leishmania braziliensis infections. J Infect Dis 2015;211:274-82.

[9] Wang H, Maeda Y, Fukutomi Y, Makino M. An in vitro model of Mycobacterium leprae induced granuloma formation. BMC Infect Dis 2013;13:279.

[10] Mendonca VA, Melo GE, Teixeira MM, Martins-Filho OA, Antunes CM, Teixeira AL. Analysis of chemokine receptors on the surface of circulating leukocytes of individuals infected with Mycobacterium leprae: preliminary results. Rev Soc Bras Med Trop 2008;41(Suppl 2):95-8.

[11] Ohkawa S. Activation of human monocytes in leprosy. Microbiol Immunol $1985 ; 29: 265-74$

[12] Rossol M, Kraus S, Pierer M, Baerwald C, Wagner U. The CD14(bright) CD16+ monocyte subset is expanded in rheumatoid arthritis and promotes expansion of the Th17 cell population. Arthritis Rheum 2012;64:671-7.

[13] Moniuszko M, Bodzenta-Lukaszyk A, Kowal K, Lenczewska D, Dabrowska M. Enhanced frequencies of $\mathrm{CD} 14++\mathrm{CD} 16+$, but not $\mathrm{CD} 14+\mathrm{CD} 16+$, peripheral blood monocytes in severe asthmatic patients. Clin Immunol 2009;130: 338-46.

[14] Novais FO, Nguyen BT, Beiting DP, Carvalho LP, Glennie ND, Passos S, et al. Human classical monocytes control the intracellular stage of Leishmania braziliensis by reactive oxygen species. J Infect Dis 2014;209:1288-96.

[15] Campos TM, Passos ST, Novais FO, Beiting DP, Costa RS, Queiroz A, et al. Matrix metalloproteinase 9 production by monocytes is enhanced by TNF and participates in the pathology of human cutaneous Leishmaniasis. PLoS Neglected Trop Dis 2014;8:e3282.

[16] Belge KU, Dayyani F, Horelt A, Siedlar M, Frankenberger M, Frankenberger B, et al. The proinflammatory $\mathrm{CD} 14+\mathrm{CD} 16+\mathrm{DR}++$ monocytes are a major source of TNF. J Immunol 2002;168:3536-42.

[17] Agrewala JN, Kumar B, Vohra H. Potential role of B7-1 and CD28 molecules in immunosuppression in leprosy. Clin Exp Immunol 1998;111:56-63.

[18] Schlienger K, Uyemura K, Jullien D, Sieling PA, Rea TH, Linsley PS, et al. B7-1, but not $\mathrm{CD} 28$, is crucial for the maintenance of the $\mathrm{CD} 4+\mathrm{T}$ cell responses in human leprosy. J Immunol 1998;161:2407-13.

[19] Santos DO, Castro HC, Bourguignon SC, Bastos OM, Rodrigues CR, Van Heuverswyn $\mathrm{H}$, et al. Expression of B7-1 co-stimulatory molecules in patients with multibacillary leprosy and reactional states. Clin Exp Dermatol 2007;32: 75-80.

[20] Spierings E, De Boer T, Zulianello L, Ottenhoff TH. The role of Schwann cells, T cells and Mycobacterium leprae in the immunopathogenesis of nerve damage in leprosy. Lepr Rev 2000:71(Suppl):S121-9.

[21] Thomas GR, Chen Z, Leukinova E, Van Waes C, Wen J. Cytokines IL-1 alpha, IL6 , and GM-CSF constitutively secreted by oral squamous carcinoma induce down-regulation of CD80 costimulatory molecule expression: restoration by interferon gamma. Cancer Immunol Immunother 2004:53:33-40.

[22] Green KA, Cook WJ, Sharpe AH, Green WR. The CD154/CD40 interaction required for retrovirus-induced murine immunodeficiency syndrome is not mediated by upregulation of the CD80/CD86 costimulatory molecules. J Virol 2002;76:13106-10.

[23] Bour-Jordan H, Esensten JH, Martinez-Llordella M, Penaranda C, Stumpf M, Bluestone JA. Intrinsic and extrinsic control of peripheral T-cell tolerance by costimulatory molecules of the CD28/B7 family. Immunol Rev 2011;241: 180-205.

[24] Lenschow DJ, Walunas TL, Bluestone JA. CD28/B7 system of T cell costimulation. Annu Rev Immunol 1996:14:233-58.

[25] Villeneuve J, Desmoulière A, Dewitte A, Bordeau N, Costet P, Bassaganyas L, et al. A role for CD154, the CD40 ligand, in granulomatous inflammation. Mediat Inflamm 2017;2017:2982879. 